\title{
Uso y abuso de sustancias psicotrópicas e internet, psicopatología e ideación suicida en adolescentes
}

\section{Substance use or abuse, internet use, psychopathology and suicidal ideation in adolescents?}

\author{
Matilde Bousoño*, Susana Al-Halabí******, Patricia Burón******, Marlen Garrido*, Eva \\ Ma Díaz-Mesa*, Gonzalo Galván*, leticia García-Alvarez**, Vladimir Carli****, Christina \\ Hoven*****, Marco Sarchiapone******, Danuta Wasserman****, Manuel Bousoño*******,

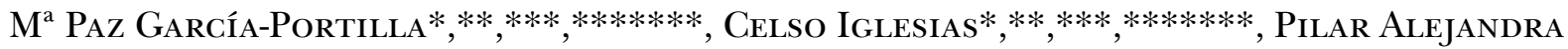 \\ SÁIZ*,**,***,*******, Julio BobeS*,**,***,*******.
}

*Área de Psiquiatría. Universidad de Oviedo. **Centro de Investigación Biomédica en Red de Salud Mental. CIBERSAM. ***Instituto de Neurociencias del Principado de Asturias. INEUROPA. **** National Centre for Suicide Research and Prevention of Mental III-Health, Karolinska Institutet, Stockholm, Sweden. *****Division of Child and Adolescent Psychiatry and Department of Psychiatry, New York State Psychiatric Institute, Columbia University, New York, NY, USA. Department of Epidemiology, Mailman School of Public Health, Columbia University, New York, NY, USA ******Department of Medicine and Health Science, University of Molise, Campobasso, Italy. National Institute for Health, Migration and Poverty, Rome, Italy. *******Servicio de Salud del Principado de Asturias. SESPA.

\section{Resumen}

El uso o abuso de sustancias o internet, la psicopatología y la ideación suicida parecen estar relacionadas. El objetivo del presente estudio es investigar la asociación en población adolescente entre consumo de sustancias potencialmente adictivas, uso inadecuado de internet, psicopatología e ideación suicida. El estudio forma parte del proyecto europeo Saving and Empowering Young Lives in Europe (SEYLE). La muestra está compuesta por 1026 adolescentes con edades comprendidas entre 14 y 16 años procedentes de 12 centros escolares públicos del Principado de Asturias (530 varones y 496 mujeres). El presente trabajo aporta la posibilidad de conocer si los datos generales del proyecto SEYLE varían en una zona relativamente aislada y socioeconómicamente en recesión.

Las tasas obtenidas de consumo de las distintas sustancias y de uso de internet fueron: a) alcohol: $11,89 \%$ en varones y 7,86\% en mujeres; b) tabaco: $4,15 \%$ y $5,44 \%$ en varones y mujeres respectivamente; c) otras drogas: 6,98\% en varones y un 4,44\% en mujeres; d) uso de internet desadaptativo o patológico: $14,53 \%$ y $20,77 \%$ en varones y mujeres respectivamente.

Se ha observado que las variables con capacidad predictiva sobre las conductas suicidas fueron: tentativas suicidas previas, síntomas depresivos, uso desadaptativo o patológico de internet, problemas con los compañeros y consumo de alcohol.

Palabras clave: Suicidio; Sustancias; Internet; Psicopatología; Adolescentes.

\begin{abstract}
Substance and Internet use or abuse, psychopathology and suicidal ideation appear to be related. The aim of this study is to investigate the association between use of psychotropic substances, inadequate Internet use, suicidal ideation and other psychopathological symptoms within the adolescent population. The present study was carried out as part of the Saving and Empowering Young Lives in Europe (SEYLE) project, funded by the European Union. The sample is composed of 1026 adolescents aged between 14 and 16 years from 12 state schools in Asturias (530 men and 496 women). This study adds to the possibility of knowing whether the SEYLE data is confirmed in a relatively isolated and recession hit province of Spain.

In the present study the following consumption rates were obtained: a) alcohol $11.89 \%$ in males and $7.86 \%$ in females; b) tobacco: $4.15 \%$ and $5.44 \%$ in males and females respectively; c) other drugs: $6.98 \%$ in males and $4.44 \%$ in females; d) maladaptive or pathological Internet use: $14.53 \%$ and $20.77 \%$ in males and females respectively.

The variables that predict suicide ideation in the logistic regression model were: previous suicide attempts, depression, maladaptive or pathological Internet use, peer problems and alcohol consumption. Keywords: Suicide; Substances; Internet; Psychopathology; Teenagers.
\end{abstract}

Recibido: Abril 2016; Aceptado: Noviembre 2016.

Enviar correspondencia a:

Julio Bobes García. Área de Psiquiatría. Facultad de Medicina. Julián Clavería 6- 3º,33006 Oviedo.

E-mail: bobes@uniovi.es 


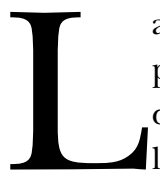

as conductas suicidas y el consumo de sustancias psicoactivas potencialmente adictivas, son dos de los problemas que mayor impacto causan en la salud pública mundial, singularmente en los jóvenes. El suicidio es la segunda causa de muerte en personas con edades entre los 15 y los 29 años (World Health Organization, 2012), y la proporción de consumo de psicótropos entre los jóvenes es alta. En 2014 el porcentaje de jóvenes que había consumido sustancias psicoactivas en el último año fue: alcohol un 76,8\%; tabaco un 31,4\%; cannabis un 25,4\%; cocaína 2,8\% y menos de un $1 \%$ otros tipos de drogas ilegales (ESTUDES 2014). Dentro del capítulo de las conductas que pueden llegar a la consideración de adicciones no relacionadas con sustancias (adicciones comportamentales), el uso excesivo de internet es un fenómeno creciente de nuestro tiempo que se da, sobre todo, en los grupos de gente joven (Fioravanti, Dettore y Casale, 2012; Seybert, 2012; Smahel, Blinka y Ledabyl, 2008).

En los últimos años, el uso patológico de internet ha trascendido la consideración de trastorno del control de impulsos (Young, 1999) para encuadrarse en las adicciones comportamentales (Griffiths, 2000), aceptando que tiene una base común con el resto de conductas adictivas (Brezing, Derevensky y Potenza, 2010; Goldstein y Volkow, 2011; Grant, Potenza, Weinstein y Gorelick, 2010; Kormas, Critselis, Janikian, Kafetzis y Tsitsika, 2011; Montag, Kirsch, Sauer, Markett y Reuter, 2012; Zhou et al., 2011). El DSM-5 (APA, 2013) incluye el juego patológico en los trastornos adictivos y el Trastorno por Juego en Internet (Internet Gaming Disorder) en el apartado condiciones para más estudios en el futuro (Sección III). No obstante, a pesar de su importancia, el manual no incluye como trastorno la adicción a internet o a las nuevas tecnologías virtuales en su conjunto (Carbonell, 2014).

Entre las personas que se inyectan drogas, el uso de sustancias como alcohol, sedantes-hipnóticos y cannabis, parece estar asociado con una mayor probabilidad de intento de suicidio posterior (Artenie et al., 2015). Las personas con dependencia de sustancias e historia de depresión tienen mayor riesgo de cometer tentativas suicidas, con independencia de si la depresión había ocurrido antes o durante el abuso de sustancias (Aharonovich, Liu, Nunes y Hasin, 2002). Otros tipos de trastornos del humor muestran la misma tendencia, por ejemplo los pacientes con Trastorno Bipolar y abuso de alcohol tienen un mayor riesgo de suicidio (Dalton, Cate-Carter, Mundo, Parikh y Kennedy, 2003; Hawton, Sutton, Haw, Sinclair y Harriss, 2005; Oquendo et al., 2010; Potash et al., 2000).

En adolescentes, la adicción a internet se asocia con el trastorno por déficit de atención e hiperactividad (Gundogar, Bakim, Ozer y Karamustafalioglu, 2012; Yoo et al., 2004), con trastornos depresivos (Andreou y Svoli, 2013; Sasmaz et al., 2014), con trastornos de ansiedad (Lee y Stapinski, 2012; Zboralski et al., 2009) y con conductas suici- das (Fernández-Villa et al., 2015; Hakala, Rimpela, Saarni y Salminen, 2006; Kim, 2012; Kim et al., 2016; Shapira, Goldsmith, Keck, Khosla y McElroy, 2000).

El estudio de la relación entre el consumo de sustancias, el uso o abuso de internet y la ideación y conductas suicidas y psicopatología (Al-Asadi, Klein y Meyer, 2015), está justificado porque podría aumentar la posibilidad de obtener una mayor comprensión de los substratos psicopatológicos que subyacen a estos fenómenos (Kaess et al., 2014).

El objetivo del presente trabajo es conocer la situación de una población joven en relación con el consumo de sustancias psicotrópicas, la ideación suicida y otros síntomas psicopatológicos, y explorar las posibles asociaciones entre estos aspectos. El trabajo se circunscribe a la Comunidad Autónoma del Principado de Asturias, una de las zonas de España con menor crecimiento en el periodo de 20002009, en el que ha sufrido una fuerte recesión socioeconómica (INE, 2010) y tiene el interés de que permite valorar si la situación socioeconómica singular puede producir datos diferenciales. También aporta la valoración del uso inadecuado de internet como adicción comportamental emergente con influencia en la ideación suicida.

\section{Método}

Estudio epidemiológico observacional, descriptivo y transversal, en el que se analizan los datos españoles del proyecto Saving and Empowering Young Lives in Europe (SEYLE) (Wasserman et al., 2012) utilizando la metodología propia del mismo (Kaess et al., 2014).

\section{Participantes}

La muestra (sub-muestra española) está compuesta por 1026 sujetos, de 12 centros educativos públicos de Oviedo, Gijón y Avilés seleccionados de forma aleatoria entre los centros de la Comunidad Autónoma de Asturias, teniendo en cuenta los criterios de inclusión y exclusión del proyecto SEYLE (Wasserman et al., 2010).

\section{Procedimiento}

En cumplimiento de las normas que rigen la investigación en población joven, previamente al inicio del estudio se obtuvo la autorización del fiscal de menores y la aprobación del Comité de Ética e Investigación Clínica del Principado de Asturias. Las autoridades escolares locales otorgaron permiso para acceder a las escuelas seleccionadas al azar y los sujetos de estudio otorgaron el asentimiento y consentimiento informado, según fuera requerido.

\section{Instrumentos}

Se utilizó un cuestionario de auto-informe estructurado que fue administrado en el aula en horario escolar y recoge datos sobre estilos de vida, conductas, valores, salud mental y suicidalidad. 
La ideación y conducta suicida fueron medidas por medio de la escala de suicidio de Paykel (PSS) (Paykel, Myers, Lindenthal y Tanner, 1974). El PSS comprende las siguientes cinco preguntas: durante las últimas 2 semanas: (i) ¿Has sentido que la vida no merece la pena?, (ii) ¿Has deseado estar muerto?, (iii) ¿Has pensado en quitarte la vida aunque realmente no lo fueras a hacer?, (iv) ¿Has llegado al punto en el que consideraras realmente quitarte la vida o hicieses planes sobre cómo lo harías?; y (v) ¿Alguna vez has intentado quitarte la vida?. Se consideró que la persona tenía pensamientos suicidas si contestaba "si" a la tercera (iii) o la cuarta (iv) cuestión del PSS. Los intentos de suicidio fueron definidos por la respuesta "si" a la última pregunta (v) del PSS.

Para la valoración del consumo de sustancias se ha utilizado el cuestionario Global School-based Student Health Survey -GSHS- (World Health Organization, 2015), estableciendo los siguientes puntos de corte: en el consumo de alcohol se ha considerado como punto de corte el consumir cualquier cantidad de alcohol dos veces o más a la semana; en el consumo de drogas, haber usado drogas ilegales al menos tres veces a lo largo de la vida y en el consumo de tabaco fumar más de diez cigarrillos al día.

El Uso Patológico de Internet se ha evaluado mediante el Young's Diagnostic Questionnaire (YDQ) (Young, 1998). La puntuación de los 8 ítems refleja ocho de los nueve criterios para el trastorno de juego en Internet en el DSM-5. En función de las respuestas al cuestionario, los sujetos fueron divididos en tres categorías de uso de Internet. Asignando un punto a cada respuesta afirmativa, se consideró que un Uso Adaptativo de Internet -UIA - correspondería a los sujetos con una puntuación entre 0 y 2 , mientras que un Uso Desadaptativo de Internet -UID- serían las puntuaciones de 3 y 4 . Una puntuación de 5 o más correspondería a un Uso Patológico de Internet -UIP- (Kaess et al., 2014).

Se utilizó el inventario de Beck (BDI-II) para evaluar los síntomas depresivos (Beck, Steer, Ball y Ranieri, 1996), considerando que una puntuación igual o mayor de 20 suponía riesgo de depresión. Para el presente estudio se ha utilizado una versión modificada, el BDI-II. El ítem "pérdida de la libido" fue retirado de la escala ya que se consideró una pregunta inapropiada para la población adolescente. La evidencia muestra que la omisión de esta pregunta no afecta a la fiabilidad o validez del instrumento (Byrne, Stewart y Lee, 2004).

Para evaluar la psicopatología se empleó el Cuestionario de Capacidades y Dificultades (SDQ) (Goodman, Meltzer y Bailey, 2003) que valora síntomas emocionales, problemas de conducta, hiperactividad/falta de atención, problemas de relación entre pares y comportamiento pro-social. Los puntos de corte seleccionados fueron: una puntuación mayor o igual que 7 para los síntomas emocionales, una puntuación mayor o igual que 5 para los problemas de conducta y una puntuación mayor o igual que 7 para la hiperactividad. En el caso de los problemas con los compa- ñeros, el punto de corte se fijó en una puntuación mayor o igual que 6 , mientras que la falta de comportamiento pro-social se definió con una puntuación menor o igual que 4 (Carli et al., 2013).

Para evaluar el bienestar subjetivo global se ha utilizado el Cuestionario de Bienestar WHO-5 (Topp, Ostergaard, Sondergaard y Bech, 2015).

\section{Análisis de datos}

Se realizó un estudio de la muestra global y desagregada por sexo. Los valores de las variables categóricas se expresaron en frecuencias y porcentajes y los de las variables continuas en medias y desviaciones estándar. En las variables categóricas la comparación entre grupos se realizó por medio del test de Chi-cuadrado o bien del test exacto de Fisher en aquellos casos en los que se encontró algún grupo de tamaño inferior a 5. En el caso de variables cuantitativas se empleó el test t de Student para la comparación de medias.

El análisis de la relación entre la ideación suicida, los comportamientos de riesgo y las características psicopatológicas de los integrantes de la muestra, se realizó a través de un modelo de regresión logística con selección hacia adelante, incluyendo como variables dependientes todas las que resultaron significativas en el análisis univariante (Tablas 3 y 4), la edad y el sexo. La significación estadística se estableció en un nivel alfa del $5 \%$.

\section{Resultados}

Se estudiaron 1026 escolares, $530(51,66 \%)$ varones y $496(48,34 \%)$ mujeres con edades comprendidas entre $14 \mathrm{y}$ 16 años de edad. La edad media de la muestra estudiada fue de 14,52 años $(\mathrm{DE}=0,70)$, sin diferencias entre los sexos.

La Tabla 1 muestra la tasa de consumo de sustancias y patrones de uso de internet en función del sexo, observándose un consumo de alcohol significativamente mayor en los varones y un uso desadaptativo o patológico de internet significativamente mayor en las mujeres.

Tabla 1. Comportamientos de riesgo en función del sexo. Consumo de sustancias (alcohol, drogas y tabaco) y uso de Internet.

\begin{tabular}{lrrrr}
\hline & $\begin{array}{r}\text { Varones } \\
(\mathbf{n = 5 3 0 )}\end{array}$ & $\begin{array}{r}\text { Mujeres } \\
(\mathbf{n = 4 9 6 )}\end{array}$ & $\mathbf{X}^{2}$ & p-valor \\
\hline Consumo de alcohol & $63(11,89 \%)$ & $39(7,86 \%)$ & 4,20 & 0,040 \\
Consumo de drogas & $37(6,98 \%)$ & $22(4,44 \%)$ & 2,61 & 0,132 \\
Consumo de tabaco & $22(4,15 \%)$ & $27(5,44 \%)$ & 0,68 & 0,410 \\
Uso de Internet & & & 4,91 & 0,027 \\
UIA & $453(85,47 \%)$ & $393(79,23 \%)$ & & \\
UID & $58(10,94 \%)$ & $78(15,73 \%)$ & & \\
UIP & $19(3,59 \%)$ & $25(5,04 \%)$ & \\
\hline
\end{tabular}

Nota. UIA: uso de Internet adaptativo, UID: uso de Internet desadaptativo y UIP: uso patológico de Internet. 
Tabla 2. Características psicopatológicas en función del sexo.

\begin{tabular}{|c|c|c|c|c|}
\hline & $\begin{array}{l}\text { Varones } \\
(n=530)\end{array}$ & $\begin{array}{l}\text { Mujeres } \\
(n=496)\end{array}$ & $\mathrm{X}^{2} / \mathrm{T}$ & p-valor \\
\hline Depresión (BDI) & $29(5,47 \%)$ & $40(8,06 \%)$ & 3,25 & 0,126 \\
\hline $\begin{array}{l}\text { Síntomas } \\
\text { emocionales (SDQ) }\end{array}$ & $9(1,70 \%)$ & $56(11,29 \%)$ & 38,13 & $<0,001$ \\
\hline $\begin{array}{l}\text { Problemas de } \\
\text { conducta (SDQ) }\end{array}$ & $32(6,04 \%)$ & $19(3,83 \%)$ & 2,20 & 0,138 \\
\hline $\begin{array}{l}\text { Hiperactividad } \\
\text { (SDQ) }\end{array}$ & $106(20,00 \%)$ & $57(11,49 \%)$ & 13,25 & $<0,001$ \\
\hline $\begin{array}{l}\text { Problemas con los } \\
\text { compañeros (SDQ) }\end{array}$ & $15(2,83 \%)$ & $14(2,82 \%)$ & 0,03 & 0,863 \\
\hline $\begin{array}{l}\text { Falta de } \\
\text { comportamiento } \\
\text { pro-social (SDQ) }\end{array}$ & $17(3,21 \%)$ & $1(0,20 \%)$ & & $<0,001$ \\
\hline Ideación suicida & $37(6,98 \%)$ & $40(8,06 \%)$ & 0,43 & 0,510 \\
\hline $\begin{array}{l}\text { Tentativas suicidas } \\
\text { previas }\end{array}$ & $11(2,08 \%)$ & $21(4,23 \%)$ & 3,9503 & 0,046 \\
\hline $\begin{array}{l}\text { Calidad de vida } \\
\text { (WHO-5) }\end{array}$ & $\begin{array}{r}68,21 \\
(\mathrm{DE}=19,16)\end{array}$ & $\begin{array}{r}64,40 \\
(D E=18,15)\end{array}$ & 3,27 & $<0,001$ \\
\hline
\end{tabular}

En cuanto a la situación psicopatológica (Tabla 2) se vio que las mujeres presentaron más síntomas emocionales y tentativas suicidas previas, mientras que los hombres puntuaron más alto en hiperactividad y falta de comportamiento pro-social en la escala SDQ. Asimismo, se registraron diferencias estadísticamente significativas en la percepción de calidad de vida según el cuestionario WHO-5 (superior en los varones).

Dividiendo a los sujetos del estudio en función de la presencia de ideación suicida (Tabla 3), se encontró asociación significativa entre el consumo de sustancias (alcohol, drogas ilegales y tabaco) y el uso desadaptativo o patológico de internet con la ideación suicida. En la tabla 4 se ve la asociación positiva entre todas las características psicopatológicas estudiadas y la ideación suicida.

En la Tabla 5 se muestran los resultados del modelo de regresión logística definido para la probabilidad de ocurrencia del evento ideación suicida. La presencia de síntomas depresivos y la existencia de tentativas suicidas previas tuvieron una capacidad predictiva grande sobre los comportamientos suicidas. Esta fue menor en el caso de problemas con los compañeros, consumo de alcohol y uso inadecuado de internet. Existen algunas variables que resultaban significativas al realizar la estadística univariante tales como el consumo de drogas, cuya relación desaparece cuando se estudia en conjunto con el efecto de las demás variables.

\section{Discusión}

En el presente estudio, realizado en adolescentes de ambos sexos de 14,52 años de edad media, se han encontrado unas tasas elevadas de consumo de sustancias y de uso des-
Tabla 3. Comportamientos de riesgo en función de la existencia de ideación suicida. Consumo de sustancias (alcohol, drogas y tabaco) y uso de Internet en hombres y mujeres.

\begin{tabular}{lrrrr}
\hline & $\begin{array}{r}\text { Ideación } \\
\text { suicida } \\
(\mathbf{n = 7 7 )}\end{array}$ & $\begin{array}{r}\text { No ideación } \\
\text { suicida } \\
(\mathbf{n = 9 4 9 )}\end{array}$ & $\mathbf{X}^{2}$ & p-valor \\
\hline Consumo de alcohol & $25(32,47 \%)$ & $77(8,11 \%)$ & 44,50 & $<0,001$ \\
Consumo de drogas & $15(19,48 \%)$ & $44(4,64 \%)$ & 26,28 & $<0,001$ \\
Consumo de tabaco & $9(11,69 \%)$ & $40(4,21 \%)$ & 7,18 & 0,007 \\
Uso de Internet & & & 590,11 & $<0,001$ \\
UIA & $37(48,05 \%)$ & $(85,25 \%)$ & & \\
UID & $25(32,47 \%)$ & 111 & & \\
UIP & $15(19,48 \%)$ & $29(3,06 \%)$ & & \\
\hline
\end{tabular}

Nota. UIA: uso de Internet adaptativo, UID: uso de Internet desadaptativo y UIP: uso patológico de Internet.

Tabla 4. Características psicopatológicas de los integrantes de la muestra en función de si presentan ideación suicida o no.

\begin{tabular}{lrrrr}
\hline & $\begin{array}{r}\text { Ideación } \\
\text { suicida } \\
(\mathbf{n = 7 7 )}\end{array}$ & $\begin{array}{r}\text { No ideación } \\
\text { suicida } \\
(\mathbf{n = 9 4 9 )}\end{array}$ & $\mathbf{X}^{2} / \mathbf{T}$ & p-valor \\
\hline $\begin{array}{l}\text { Depresión (BDI) } \\
\text { Síntomas } \\
\text { emocionales (SDQ) }\end{array}$ & $22(28,57 \%)$ & $43(4,53 \%)$ & 65,37 & $<0,001$ \\
$\begin{array}{l}\text { Problemas de } \\
\text { conducta (SDQ) }\end{array}$ & $16(20,78 \%)$ & $35(3,69 \%)$ & 40,50 & $<0,001$ \\
$\begin{array}{l}\text { Hiperactividad } \\
\text { (SDQ) }\end{array}$ & $20(25,97 \%)$ & $143(15,07 \%)$ & 55,49 & 0,018 \\
$\begin{array}{l}\text { Problemas con los } \\
\text { compañeros (SDQ) }\end{array}$ & $10(12,99 \%)$ & $19(2,00 \%)$ & 27,42 & $<0,001$ \\
$\begin{array}{l}\text { Falta de } \\
\text { comportamiento } \\
\text { pro-social (SDQ) }\end{array}$ & $5(6,49 \%)$ & $13(1,37 \%)$ & 8,08 & 0,004 \\
$\begin{array}{l}\text { Tentativas suicidas } \\
\text { previas }\end{array}$ & $18(23,38 \%)$ & $14(1,48 \%)$ & 105,93 & $<0,001$ \\
$\begin{array}{l}\text { Calidad de vida } \\
\text { (WHO-5) }\end{array}$ & $46,03(19,43)$ & $68,02(17,73)$ & 9,61 & $<0,001$ \\
\hline
\end{tabular}

Tabla 5. Modelo de regresión logística de ideación suicida.

\begin{tabular}{|c|c|c|c|c|}
\hline Variable & B & O.R. & IC $95 \%$ & p \\
\hline Constante & $-2,03$ & 0,13 & $(0,05 ; 0,38)$ & $<0,001$ \\
\hline Uso de Internet & & & & $<0,001$ \\
\hline UID vs UIA & 1,09 & 2,37 & $(1,19 ; 4,76)$ & \\
\hline UIP vs UID & 1,37 & 2,56 & $(0,95 ; 6,92)$ & \\
\hline Consumo de alcohol & 1,47 & 3,44 & $(1,67 ; 7,07)$ & $<0,001$ \\
\hline Depresión (BDI) & 2,45 & 9,26 & $(4,60 ; 18,64)$ & $<0,001$ \\
\hline $\begin{array}{l}\text { Problemas con los } \\
\text { compañeros (SDQ) }\end{array}$ & 1,92 & 3,88 & $(1,22 ; 12,37)$ & 0,029 \\
\hline $\begin{array}{l}\text { Tentativas suicidas } \\
\text { previas }\end{array}$ & 2,52 & 8,45 & $(3,31 ; 21,55)$ & $<0,001$ \\
\hline $\begin{array}{l}\text { Calidad de vida } \\
\text { (WHO-5) }\end{array}$ & $-0,03$ & 0,97 & $(0,95 ; 0,99)$ & $<0,001$ \\
\hline
\end{tabular}


adaptativo o patológico de internet, que son preocupantes por las consecuencias negativas para la salud física y psicológica de los jóvenes (Fernández-Villa et al., 2013).

Se observan diferencias en el perfil de consumo de sustancias, que son congruentes con los datos del proyecto SEYLE en su conjunto (Carli et al., 2014), pero inferiores a los datos de otros estudios españoles realizados sobre poblaciones de más edad. La encuesta ESTUDES (ESTUDES 2014) realizada en estudiantes de secundaria (14-18 años) o un estudio realizado sobre estudiantes universitarios (Hernández-Serrano, Font-Mayolas y Gras, 2015) encuentran tasas de consumo de todas las sustancias muy superiores; además, en el caso de los de mayor edad (universitarios) el tabaco fue la sustancia más consumida seguida del cannabis. La edad media es un elemento a considerar (14 años en el presente estudio), ya que el inicio del consumo de alcohol o tabaco en edades tempranas se asocia con consumos regulares posteriores e incrementa el riesgo de desarrollar otros trastornos por consumo de sustancias o una dependencia y se asocia también con el juego problema (Míguez y Becoña, 2015; Motos, Cortés, Giménez y Cadaveira, 2015). En cuanto al uso de internet, los datos son similares a la muestra europea, excepto en el caso del UIP que en la muestra europea es más frecuente en varones ( $5,2 \%$ en hombres y $3,8 \%$ en mujeres). Las condiciones socioeconómicas y la falta de apoyo social y familiar pueden ser la causa de variaciones en el riesgo de UIP (Durkee et al., 2012, 2016).

Las tasas de síntomas psicopatológicos son altas teniendo en cuenta la edad de la población estudiada. Llama la atención que la prevalencia de trastornos hiperactivos en varones $(20 \%)$ dobla la tasa de la muestra europea (alrededor del 10\%), no así en las mujeres. Las cifras de prevalencia del Trastorno por Déficit de Atención e Hiperactividad obtenidas por otros estudios oscilan en torno al 5\% (Polanczyk, de Lima, Horta, Biederman y Rohde, 2007; Schlack, Mauz, Hebebrand y Holling, 2014; Willcutt, 2012). Esta diferencia tan importante, podría plantear dudas respecto a la validez de la escala utilizada (SDQ).

En el caso de la ideación suicida, las tasas de la muestra europea $(21,2 \%$ en varones y $35,4 \%$ en mujeres) casi cuadriplican las de la muestra española. A pesar de la dificultad de determinar los condicionantes de la ideación y conducta suicida, probablemente las diferencias socio-culturales existentes entre España y el resto de la Unión Europea justifiquen, al menos en parte, la diferencia. La cultura del suicidio ha penetrado más en otras sociedades y aunque en España se ha observado un incremento en los últimos años, las cifras de suicidalidad son inferiores a las de los países de nuestro entorno (Alvaro-Meca, Kneib, Gil-Prieto y Gil de, 2013; Kolves y De Leo, 2016).

Los datos del presente estudio, en general, confirman la asociación entre consumo de sustancias psicotrópicas potencialmente adictivas, uso inadecuado de internet, psi- copatología e ideación suicida que podría fundamentarse en elementos de personalidad o mecanismos neurobiológicos comunes (Albert, Rosso, Maina y Bogetto, 2008; Schoevers, Deeg, Van y Beekman, 2005; Sher, 2006). No obstante, se encuentran algunas diferencias, sobre todo en la falta de asociación significativa entre ideación suicida y consumo de sustancias diferentes del alcohol, o en el casi nulo efecto de la calidad de vida sobre la suicidalidad.

El efecto del uso de internet sobre la suicidalidad es confuso. Por un lado se considera que incrementa el riesgo de conducta suicida, ya que facilita la interacción con otras personas con intenciones suicidas y está demostrado que la exposición a estas conductas a través de internet se asocia al empleo de métodos más peligrosos de autolesión. También puede ser utilizado para el ciberbullying (Collings, Fortune, Steers, Currey y Hawton, 2011). Por otro lado, internet podría proteger de la ideación suicida si se utiliza como fuente de apoyo emocional o como instrumento de mejora de las estrategias de afrontamiento (Daine et al., 2013). En cualquier caso, si se habla de uso inadecuado de internet, la balanza parece decantarse hacia los efectos negativos y su uso en general está asociado a un mayor riesgo de autolesiones, ideación suicida y depresión (Madge et al., 2011; O’Connor, Rasmussen y Hawton, 2012).

El presente estudio presenta algunas limitaciones, entre las que está el tamaño de la muestra que, aunque amplia, no permite establecer asociaciones entre variables poco frecuentes como el consumo de drogas o realizar análisis desagregado por grupos de edad.

\section{Conclusiones}

El presente estudio ha encontrado una prevalencia relativamente alta de consumo de substancias psicotrópicas, uso desadaptativo o patológico de internet, ideación suicida y síntomas psicopatológicos en una muestra de adolescentes jóvenes.

Las variables que predicen la ideación suicida con un efecto relevante son: tentativas suicidas previas, presencia de síntomas depresivos, uso desadaptativo o patológico de internet, consumo de alcohol y problemas con los compañeros.

\section{Conflicto de intereses}

Los autores declaran que no existe conflicto de interés.

\section{Reconocimientos}

El Proyecto SEYLE ha sido financiado por la Unión Europea a través del Séptimo Programa Marco (FP7), mediante acuerdo de subvención número HEALTH-F2.2009-223091. Los autores desean agradecer a todos los investigadores y personal que participa en el Programa SEYLE. También ha 
sido en parte subvencionado por el Ministerio Español de Economía y Competitividad, Instituto de Salud Carlos III a través del Centro de Investigación en Red de Salud Mental (CIBERSAM)

\section{Referencias}

Aharonovich, E., Liu, X., Nunes, E. y Hasin, D. S. (2002). Suicide attempts in substance abusers: effects of major depression in relation to substance use disorders. American Journal of Psychiatry, 159, 1600-1602. doi:10.1176/ appi.ajp.159.9.1600.

Al-Asadi, A. M., Klein, B. y Meyer, D. (2015). Multiple comorbidities of 21 psychological disorders and relationships with psychosocial variables: a study of the online assessment and diagnostic system within a web-based population. Journal of Medical Internet Research, 17, e55. doi:10.2196/jmir.4143.

Albert, U., Rosso, G., Maina, G. y Bogetto, F. (2008). Impact of anxiety disorder comorbidity on quality of life in euthymic bipolar disorder patients: differences between bipolar I and II subtypes. Journal of Affective Disorders, 105, 297-303. doi:10.1016/j.jad.2007.05.020.

Alvaro-Meca, A., Kneib, T., Gil-Prieto, R. y Gil de, M. A. (2013). Epidemiology of suicide in Spain, 1981-2008: a spatiotemporal analysis. Public Health, 127, 380-385. doi:10.1016/j.puhe.2012.12.007.

Andreou, E. y Svoli, H. (2013). The association between internet user characteristics and dimensions of internet addiction among Greek adolescents. International Journal of Mental Health and Addiction, 11, 139-148. doi:10.1007/ s11469-012-9404-3.

APA, American Psychiatric Association (2013). Diagnostic and statistical manual of mental disorders (5th Edition). Washington, DC: American Psychiatric Association.

Artenie, A. A., Bruneau, J., Roy, E., Zang, G., Lesperance, F., Renaud, J.,... Jutras-Aswad, D. (2015). Licit and illicit substance use among people who inject drugs and the association with subsequent suicidal attempt. Addiction, 110, 1636-1643. doi:10.1111/add.13030.

Beck, A. T., Steer, R. A., Ball, R. y Ranieri, W. (1996). Comparison of Beck Depression Inventories -IA and -II in psychiatric outpatients. Journal of Personality Assessment, 67, 588-597. doi:10.1207/s15327752jpa670313.

Brezing, C., Derevensky, J. L. y Potenza, M. N. (2010). Non-substance-addictive behaviors in youth: pathological gambling and problematic Internet use. Child and Adolescent Psychiatric Clinics of North America, 19, 625-641. doi:10.1016/j.chc.2010.03.012.

Byrne, B. M., Stewart, S. M. y Lee, P. W. H. (2004). Validating the Beck depresión inventory-II for Hong Kong community adolescents. International Journal of Testing, 4, 199-216. doi:10.1207/s15327574ijt0403_1.
Carbonell, X. (2014). La adicción a los videojuegos en el DSM-5. Adicciones, 26, 91-95.

Carli, V., Hoven, C. W., Wasserman, C., Chiesa, F., Guffanti, G., Sarchiapone, M.,... Wasserman, D. (2014). A newly identified group of adolescents at "invisible" risk for psychopathology and suicidal behavior: findings from the SEYLE study. World Psychiatry, 13, 78-86. doi:10.1002/ wps.20088.

Carli, V., Wasserman, C., Wasserman, D., Sarchiapone, M., Apter, A., Balazs, J.,... Hoven, C. W. (2013). The saving and empowering young lives in Europe (SEYLE) randomized controlled trial (RCT): methodological issues and participant characteristics. Bio Medical Central Public Health, 13, 479. doi:10.1186/1471-2458-13-479.

Collings, S. C., Fortune, S., Steers, D., Currey, N. y Hawton, K. (2011). Media influences on suicidal behaviour: An interview study of young people in New Zealand. Recuperado de http://www.tepou.co.nz/uploads/files/ resource-assets/media-influences-on-suicidal-behaviour. pdf.

Daine, K., Hawton, K., Singaravelu, V., Stewart, A., Simkin, S. y Montgomery, P. (2013). The power of the web: a systematic review of studies of the influence of the internet on self-harm and suicide in young people. PLoS ONE 8(10): e77555. doi:10.1371/journal.pone.0077555. eCollection 2013.

Dalton, E. J., Cate-Carter, T. D., Mundo, E., Parikh, S. V. y Kennedy, J. L. (2003). Suicide risk in bipolar patients: the role of co-morbid substance use disorders. Bipolar Disorders, 5, 58-61.

Durkee, T., Carli, V., Floderus, B., Wasserman, C., Sarchiapone, M., Apter, A.,... Wasserman, D. (2016). Pathological Internet Use and Risk-Behaviors among European Adolescents. International Journal of Environmental Research and Public Health, 13. doi:10.3390/ijerph13030294.

Durkee, T., Kaess, M., Carli, V., Parzer, P., Wasserman, C., Floderus, B.,... Wasserman, D. (2012). Prevalence of pathological internet use among adolescents in Europe: demographic and social factors. Addiction, 107, 22102222. doi:10.1111/j.1360-0443.2012.03946.x.

ESTUDES (2014). Encuesta sobre uso de drogas en enseñanzas secundarias en España (2014). Madrid: Ministerio de Sanidad, Servicios Sociales e Igualdad. Recuperado de http://www.pnsd.msssi.gob.es/profesionales/ sistemasInformacion/sistemaInformacion/pdf/2016_ ESTUDES_2014-2015.pdf.

Fernández-Villa, T., Alguacil, J., Almaraz, A., Cancela, J. M., Delgado-rodríguez, M., García-Martín, M.,... Martín, V. (2015). Uso problemático de internet en estudiantes universitarios: factores asociados y diferencias de género. Adicciones, 27, 265-275. doi:10.20882/adicciones.27.4.

Fernández-Villa, T., Alguacil, J., Ayán, C., Bueno, A., Cancela, J. M., Capelo, R.,... Martín, V. (2013). UNIHCOS 
Project: dynamic cohort of Spanish college students to the study of drug and other addictions. Revista Española de Salud Pública, 87, 575-85. doi:10.4321/S113557272013000600003.

Fioravanti, G., Dettore, D. y Casale, S. (2012). Adolescent Internet addiction: testing the association between self-esteem, the perception of Internet attributes, and preference for online social interactions. Cyberpsychology, Behavavior and Social Networking, 15, 318-323. doi:10.1089/cyber.2011.0358.

Goldstein, R. Z. y Volkow, N. D. (2011). Dysfunction of the prefrontal cortex in addiction: neuroimaging findings and clinical implications. Nature Reviews Neuroscience, 12, 652-669. doi:10.1038/nrn3119,

Goodman, R., Meltzer, H. y Bailey, V. (2003). The Strengths and Difficulties Questionnaire: a pilot study on the validity of the self-report version. International Review of Psychiatry, 15, 173-177. doi:10.1080/0954026021000046 137.

Grant, J. E., Potenza, M. N., Weinstein, A. y Gorelick, D. A. (2010). Introduction to behavioral addictions. American Journal of Drug and Alcohol Abuse, 36, 233-241. doi:10.310 9/00952990.2010.491884.

Griffiths, M. (2000). Internet addiction- Time to be taken seriously? Addiction Research and Theory, 8, 413-418. doi:10.3109/16066350009005587.

Gundogar, A., Bakim, B., Ozer, O. A. y Karamustafalioglu, O. (2012). P-32- The association between internet addiction, depression and ADHD among high school students. European Psychiary, 27. doi:10.1016/S09249338(12)74199-8.

Hakala, P. T., Rimpela, A. H., Saarni, L. A. y Salminen, J. J. (2006). Frequent computer-related activities increase the risk of neck-shoulder and low back pain in adolescents. European Journal of Public Health, 16, 536-541. doi:10.1093/eurpub/ck1025.

Hawton, K., Sutton, L., Haw, C., Sinclair, J. y Harriss, L. (2005). Suicide and attempted suicide in bipolar disorder: a systematic review of risk factors. Journal of Clinical Psychiatry, 66, 693-704.

Hernández-Serrano, O., Font-Mayolas, S. y Gras, M. E. (2015). Policonsumo de drogas y su relación con el contexto familiar y social en jóvenes universitarios. Adicciones, 27, 205-213. doi:10.20882/adicciones.27.3.

Instituto Nacional de Estadística (INE) (2010). Contabilidad Regional de España. Base 2000. Producto Interior Bruto regional. Serie 2000-2009. Recuperado de http:/ / www.ine.es/prensa/np640.pdf.

Kaess, M., Durkee, T., Brunner, R., Carli, V., Parzer, P., Wasserman, C.,... Wasserman, D. (2014). Pathological Internet use among European adolescents: psychopathology and self-destructive behaviours. European Child $\mathcal{E} \mathcal{F}^{2}$ Adolescent Psychiatry, 23, 1093-1102. doi:10.1007/s00787-0140562-7.
Kim, J. Y. (2012). The nonlinear association between Internet using time for non-educational purposes and adolescent health. Journal of Preventive Medicine and Public Health, 45, 37-46. doi:10.3961/jpmph.2012.45.1.37.

Kim, N. R., Hwang, S. S., Choi, J. S., Kim, D. J., Demetrovics, Z., Király, O.,... Choi, S. W. (2016). Characteristics and Psychiatric Symptoms of Internet Gaming Disorder among Adults Using Self-Reported DSM-5 Criteria. Psychiatry Investigation 13, 58-66. doi: 10.4306/ pi.2016.13.1.58.

Kolves, K. y De Leo, D. (2016). Adolescent Suicide Rates Between 1990 and 2009: Analysis of Age Group 15-19 Years Worldwide. Journal of Adolescent Health, 58, 69-77. doi:10.1016/j.jadohealth.2015.09.014.

Kormas, G., Critselis, E., Janikian, M., Kafetzis, D. y Tsitsika, A. (2011). Risk factors and psychosocial characteristics of potential problematic and problematic internet use among adolescents: a cross-sectional study. Bio Medical Central Public Health, 11, 595. doi:10.1186/1471-2458-11595.

Lee, B. W. y Stapinski, L. A. (2012). Seeking safety on the internet: relationship between social anxiety and problematic internet use. Journal of Anxiety Disorders, 26, $197-$ 205. doi:10.1016/j.janxdis.2011.11.001.

Madge, N., Hawton, K., McMahon, E. M., Corcoran, P., De, L. D., de Wilde, E. J.,... Arensman, E. (2011). Psychological characteristics, stressful life events and deliberate self-harm: findings from the Child \& Adolescent Selfharm in Europe (CASE) Study. European Child $\mathcal{E}$ Adolescent Psychiatry, 20, 499-508. doi:10.1007/s00787-0110210-4.

Míguez, M. C. y Becoña, E. (2015). ¿El consumo de cigarrillos y alcohol se relaciona con el consumo de cánnabis y el juego problema en adolescentes españoles?. Adicciones, 27, 8-16. doi: http://dx.doi.org/10.20882/adicciones.27.1.

Montag, C., Kirsch, P., Sauer, C., Markett, S. y Reuter, M. (2012). The role of the CHRNA4 gene in Internet addiction: a case-control study. Journal of Addiction Medicine, 6, 191-195. doi:10.1097/ADM.0b013e31825ba7e7.

Motos, P., Cortés, M. T., Giménez, J. A. y Cadaveira, F. (2015). Predictores del consumo semanal de alcohol y sus consecuencias asociadas en universitarios consumidores intensivos de alcohol. Adicciones, 27, 119-131. doi:10.20882/adicciones.27.2.

O’Connor, R. C., Rasmussen, S. y Hawton, K. (2012). Distinguishing adolescents who think about self-harm from those who engage in self-harm. British Journal of Psychiatry, 200, 330-335. doi:10.1192/bjp.bp.111.097808.

Oquendo, M. A., Currier, D., Liu, S. M., Hasin, D. S., Grant, B. F. y Blanco, C. (2010). Increased risk for suicidal behavior in comorbid bipolar disorder and alcohol use disorders: results from the National Epidemiologic Survey on Alcohol and Related Conditions (NESARC). 
Journal of Clinical Psychiatry, 71, 902-909. doi:10.4088/ JCP.09m05198gry.

Paykel, E. S., Myers, J. K., Lindenthal, J. J. y Tanner, J. (1974). Suicidal feelings in the general population: a prevalence study. British Journal of Psychiatry, 124, 460469.

Polanczyk, G., de Lima, M. S., Horta, B. L., Biederman, J. y Rohde, L. A. (2007). The worldwide prevalence of ADHD: a systematic review and metaregression analysis. American Journal of Psychiatry, 164, 942-948. doi:10.1176/ ajp.2007.164.6.942.

Potash, J. B., Kane, H. S., Chiu, Y. F., Simpson, S. G., MacKinnon, D. F., McInnis, M. G.,... DePaulo, J. R. (2000). Attempted suicide and alcoholism in bipolar disorder: clinical and familial relationships. American Journal of Psychiatry, 157, 2048-2050. doi:10.1176/appi. aip.157.12.2048.

Sasmaz, T., Oner, S., Kurt, A. O., Yapici, G., Yazici, A. E., Bugdayci, R.,... Sis, M. (2014). Prevalence and risk factors of Internet addiction in high school students. European Journal of Public Health, 24, 15-20. doi:10.1093/ eurpub/ckt051.

Schlack, R., Mauz, E., Hebebrand, J. y Holling, H. (2014). Has the prevalence of parent-reported diagnosis of attention deficit hyperactivity disorder (ADHD) in Germany increased between 2003-2006 and 2009-2012? Results of the KiGGS-study: first follow-up (KiGGS Wave 1). Bundesgesundheitsblatt, Gesundheitsforschung, Gesundheitsschutz, 57, 820-829. doi:10.1007/s00103-014-1983-7.

Schoevers, R. A., Deeg, D. J., Van, T. W. y Beekman, A. T. (2005). Depression and generalized anxiety disorder: co-occurrence and longitudinal patterns in elderly patients. American Journal of Geriatric Psychiatry, 13, 31-39. doi:10.1176/appi.ajqp.13.1.31.

Seybert, H. (2012). Internet use in households and by individuals in 2012. Recuperado de http://ec.europa.eu/eurostat/documents/3433488/5585460/ KSSF12050EN.PDF/39000dab-e2b7-49b2-bc4b-6aadObf01279.

Shapira, N. A., Goldsmith, T. D., Keck, P. E., Jr., Khosla, U. M. y McElroy, S. L. (2000). Psychiatric features of individuals with problematic internet use. Journal of Affective Disorders, 57, 267-272.

Sher, L. (2006). Risk and protective factors for suicide in patients with alcoholism. Scientific World Journal, 6, 14051411. doi:10.1100/tsw.2006.254.

Smahel, D., Blinka, L. y Ledabyl, O. (2008). Playing MMORPGs: connections between addiction and identifying with a character. Cyberpsychology $\mathcal{E}$ Behavior, 11, 715-718. doi:10.1089/cpb.2007.0210.

Topp, C. W., Ostergaard, S. D., Sondergaard, S. y Bech, P. (2015). The WHO-5 Well-Being Index: a systematic review of the literature. Psychotherapy and Psychosomatics, 84, 167-176. doi:10.1159/000376585.
Wasserman, C., Hoven, C. W., Wasserman, D., Carli, V., Sarchiapone, M., Al-Halabi, S.,... Postuvan, V. (2012). Suicide prevention for youth-a mental health awareness program: lessons learned from the Saving and Empowering Young Lives in Europe (SEYLE) intervention study. Bio Medical Central Public Health, 12, 776. doi:10.1186/14712458-12-776.

Wasserman, D., Carli, V., Wasserman, C., Apter, A., Balazs, J., Bobes, J.,... Hoven, C. W. (2010). Saving and empowering young lives in Europe (SEYLE): a randomized controlled trial. Bio Medical Central Public Health, 10, 192. doi:10.1186/1471-2458-10-192.

Willcutt, E. G. (2012). The prevalence of DSM-IV attention-deficit/hyperactivity disorder: a meta-analytic review. Neurotherapeutics, 9, 490-499. doi:10.1007/s13311012-0135-8.

World Health Organization (2012). Prevención del suicidio: un imperativo global. Recuperado de http://www.who. int/mental_health/suicide-prevention/es/.

World Health Organization (2015). Global School-Based Student Health Survey. Recuperado de http://www. who.int/chp/gshs/GSHS_Questionnaire_Core_2009_ Spanish.pdf?ua=1.

Yoo, H. J., Cho, S. C., Ha, J., Yune, S. K., Kim, S. J., Hwang, J.,... Lyoo, I. K. (2004). Attention deficit hyperactivity symptoms and internet addiction. Psychiatry and Clinical Neurosciences, 58, 487-494. doi:10.1111/j.14411819.2004.01290.x.

Young, K. S. (1998). Internet addiction: the emergence of a new clinical disorder. Cyberpsychology Behavior, 1:237244. doi: 10.1089/cpb.1998.1.237.

Young, K. S. (1999). Internet addiction: symptoms, evaluation and treatment. Innovations in clinical practice: a sourcebook. 17, 19-31. Recuperado de http://netaddiction.com/articles/symptoms.pdf.

Zboralski, K., Orzechowska, A., Talarowska, M., Darmosz, A., Janiak, A., Janiak, M.,... Galecki, P. (2009). The prevalence of computer and Internet addiction among pupils. Postepy Hig Med Dosw(Online.), 63, 8-12. Recuperado de http:/ / www.phmd.pl/fulltxt.php?ICID=878550.

Zhou, Y., Lin, F. C., Du, Y. S., Qin, L. D., Zhao, Z. M., Xu, J. R.,... Lei, H. (2011). Gray matter abnormalities in Internet addiction: a voxel-based morphometry study. European Journal of Radiology, 79, 92-95. doi:10.1016/j. ejrad.2009.10.025. 